\title{
Organotypic brain slices: a model to study the neurovascular unit micro-environment in epilepsies
}

\author{
Mélanie Morin-Brureau ${ }^{1,2}$, Frédéric De Bock $^{1}$ and Mireille Lerner-Natoli ${ }^{{ }^{*}}$
}

\begin{abstract}
Background: It is now recognized that the neuro-vascular unit (NVU) plays a key role in several neurological diseases including epilepsy, stroke, Alzheimer's disease, multiple sclerosis and the development of gliomas. Most of these disorders are associated with NVU dysfunction, due to overexpression of inflammatory factors such as vascular endothelial growth factor (VEGF). Various in vitro models have been developed previously to study the micro-environment of the blood-brain barrier (BBB). However none of these in vitro models contained a complete complement of NVU cells, nor maintained their interactions, thus minimizing the influence of the surrounding tissue on the BBB development and function. The organotypic hippocampal culture $(\mathrm{OHC})$ is an integrative in vitro model that allows repeated manipulations over time to further understand the development of cell circuits or the mechanisms of brain diseases.

Methods/design: OHCs were cultured from hippocampi of 6-7 day-old Sprague Dawley rats. After 2 weeks in culture, seizures were induced by application of kainate or bicuculline into culture medium. The regulation of BBB integrity under physiological and pathological conditions was evaluated by immunostaining of the main tight junction (TJ) proteins and of the basal membrane of microvessels. To mimic or prevent BBB disassembly, we used diverse pro- or anti-angiogenic treatments.

Discussion: This study demonstrates that NVU regulation can be investigated using OHCs. We observed in this model system an increase in vascularization and a down-regulation of TJ proteins, similar to the vascular changes described in a chronic focus of epileptic patients, and in rodent models of epilepsy or inflammation. We observed that Zonula occludens-1 (ZO-1) protein disappeared after seizures associated with neuronal damage. In these conditions, the angiopoeitin-1 system was down-regulated, and the application of $\mathrm{r}$-angiopoeitin-1 allowed TJ re-assembly. This article demonstrates that organotypic culture is a useful model to decipher the links between epileptic activity and vascular damage, and also to investigate NVU regulation in diverse neurological disorders.
\end{abstract}

Keywords: Neurovascular unit (NVU), Blood-brain barrier (BBB), Organotypic brain slices, Epilepsy

\section{Background}

Homeostatic maintenance is essential for proper cerebral function. The blood vessel- and non-vascular cells (neurons and glial cells) in the brain form the neurovascular unit (NVU) [1]. The NVU plays an important role in brain maintenance via cellular interactions between microvessels and parenchyma. Under physiological conditions, the NVU regulates nutrient supply, vascular growth, hemodynamics,

\footnotetext{
* Correspondence: mireille.lerner-natoli@igf.cnrs.fr

'Institut de Génomique Fonctionnelle, CNRS UMR5203, INSERM U661,

Université Montpellier 1, 2, Montpellier, France

Full list of author information is available at the end of the article
}

toxin elimination and brain protection. Adherens junctions (AJs) and tight junctions (TJs) reduce the paracellular flux across brain endothelium, whereas specific transporters and receptors carry glucose, amino-acids, nucleosides, organic anions, large amino-acids, transferrin, lipo-proteins and drugs into the brain. Conversely, pathological stimuli that increase blood-brain barrier (BBB) permeability perturb brain homeostasis. The leakage of ions, water, and serum proteins into the parenchyma modifies oncotic pressure and ionic concentrations, while leukocyte extravasation triggers immune and inflammatory responses. This imbalance leads to abnormal neuronal activity or toxicity.

\section{Biomed Central}

(c) 2013 Morin-Brureau et al.; licensee BioMed Central Ltd. This is an Open Access article distributed under the terms of the Creative Commons Attribution License (http://creativecommons.org/licenses/by/2.0), which permits unrestricted use, distribution, and reproduction in any medium, provided the original work is properly cited. 
In excitable brain structures such as the hippocampus and cerebral cortex these features induce seizures. In several CNS structures, increased BBB permeability participates in or worsens neurological disorders like Alzheimer's disease, multiple sclerosis or chronic epilepsy [2-5].

Modeling the NVU in vitro has furthered the understanding of selective mechanisms which regulate permeability, toxin elimination, nutrient supply, brain protection and homeostasis regulation. Several in vitro cell-based BBB models have previously been developed but were unable to fully recapitulate all known features of the BBB $[6,7]$. Despite the conservation of endothelial cell properties ex vivo, their isolation from multicellular blood vessels is methodologically difficult [8]. The endothelial cell monolayer is one of the most commonly used in vitro models; however, it only represents a simplified view of the BBB. This simplification reduces the interactions with other cell types, which are essential for BBB maintenance $[9,10]$. The co-culture of astrocytes and endothelial cells is the most validated cell-based BBB model. This model contains TJs, transporters, ionic channels and high transendothelial electrical resistance (TEER) necessary for a suitable model. However the absence of other cell types such as pericytes is a limitation in dynamic studies of the NVU, including vasomodulation [11]. To counteract the lack of pericytes, the tri-culture has been developed using endothelial cells, pericyte and astrocytes cell lines. In this system, all cell types are necessary for the adequate localization of TJs and transporter functions [12]. This model can be modified depending on the research objectives, using leukocytes or neurons as the third cell type $[13,14]$. The tri-culture is currently one of the most representative in vitro models to study $\mathrm{BBB}$ regulation in humans [15].

Clearly, BBB models should contain most or all cellular and molecular players of the NVU and take into account the different environmental factors. Thirty years ago, Gähwiler et al. described an integrative model to study interactions between cell types in brain slices maintained in culture [16]. This model was simplified by growing organotypic brain slices on a membrane surface [17]. These slices maintain all cell types and their interactions for 2 weeks and were mainly used to study the activity of neural cells under diverse physiologic and pathologic conditions $[18,19]$.

In 2003, it was shown for the first time that, despite the absence of blood flow in organotypic cortical slices, microvessels were present and able to respond to angiogenic stimuli like acidosis or hyperthermia [20]. Furthermore, microvessels preserved within organotypic slices respond to experimental seizures. We have used this in vitro model to study the effects of seizure-like activity on the NVU. We chose slices of rat hippocampus, since the corresponding structure in the human brain is involved in temporal lobe epilepsy (TLE). We found that kainate-induced epileptiform activities induced vascular changes in organotypic slices including angiogenesis and $\mathrm{BBB}$ alteration, similar to those reported in human intractable TLE and in vivo models [21,22].

\section{Methods/ design \\ Organotypic brain slices}

Organotypic hippocampal slices (OHCs) were prepared and cultured according to Stoppini et al. [17]. The brains of 6-7 day-old Sprague Dawley rats were removed after cold anesthesia and hippocampi were rapidly dissected under aseptic conditions in a dissection medium containing 50\% HBSS, 50\% Opti-MEM, penicillin 25 units $/ \mathrm{ml}$, streptomycin $25 \mu \mathrm{g} / \mathrm{ml}$ (Life technologies, Grand Island, NY, USA). Then transverse sections $(400 \mu \mathrm{m})$ were obtained using a tissue chopper. Ten slices were placed on a $30 \mathrm{~mm}$ porous membrane (Millipore, Billerica MA, USA) and kept in $100 \mathrm{~mm}$ diameter Petri dishes filled with $5 \mathrm{ml}$ of culture medium composed of $25 \%$ heat-inactivated horse serum, 25\% HBSS, 50\% Opti-MEM, penicillin 25 units/ml, streptomycin $25 \mu \mathrm{g} / \mathrm{ml}$ (Life technologies). Cultures were maintained in a humidified incubator at $35^{\circ} \mathrm{C}$ and $5 \% \mathrm{CO}_{2}$. One week later, cultures were transferred in defined medium composed of 25\% B27 supplemented neurobasal medium, 25\% HBSS, 50\% Opti-MEM, penicillin 25 units $/ \mathrm{ml}$, streptomycin $25 \mu \mathrm{g} / \mathrm{ml}$ (Life technologies). All animal procedures were conducted in accordance with the European Communities Council Directive of November 24, 1986 (86/6 09/EEC), and approved by the French Ministry of Agriculture (Authorization No. 34178, ML-N).

\section{Induction of "in vitro seizure"}

After 2 weeks, membranes were transferred to 6-well plates, each well filled with $1 \mathrm{ml}$ defined culture medium. To induce seizures, slices were treated with $25 \mu \mathrm{M}$ kainate (Sigma-Aldrich, Saint-Louis, MO, USA) for $1 \mathrm{~h}$ or with $10 \mu \mathrm{M}$ of bicuculline (Sigma-Aldrich) for $10 \mathrm{~min}$. Control slices received no treatment. Slices were then transferred in a bicuculline-free or kainate free-defined culture medium during the recovery period (2, 12 and $24 \mathrm{~h})$.

\section{Treatments}

\section{Recombinant proteins}

The rat recombinant vascular endothelial growth factor (rrVEGF, R\&D systems, Minneapolis, MN, USA) was added into culture medium for $24 \mathrm{~h}$ at $2 \mathrm{ng} / \mathrm{ml}$. The rhAngiopoeitin-1 (rhAng-1, R\&D systems) was added 4 $\mathrm{h}$ after seizure induction for $24 \mathrm{~h}$ at $400 \mathrm{ng} / \mathrm{ml}$. LPS (100 $\mathrm{ng} / \mathrm{ml}$, Sigma-Aldrich) was added to the culture medium for $24 \mathrm{~h}$. 
Morphological study of vascularization and tight junctions Immunostaining Slices were fixed in 4\% PFA for 30 min and stored at $4^{\circ} \mathrm{C}$ in PBS containing $0.1 \% \mathrm{NaNO}_{3}$. To evaluate vascular density and zonula occludens- 1 (ZO-1) expression, immunohistochemistry was carried out on free-floating whole slices. After pre-incubation in a PBS solution containing $10 \%$ goat serum and $0.1 \%$ Triton for $2 \mathrm{~h}$ at room temperature, slices were incubated for $48 \mathrm{~h}$ at $4^{\circ} \mathrm{C}$ with mouse or rabbit anti-laminin (Chemicon, Temecula CA, USA, 2E8, 1/ 3000 or Sigma-Aldrich, L9393 1/4000, rabbit anti-ZO1 (Zymed, San Fransisco, CA, USA, 61-7300 1/200), Goat anti-VEGF (Santa-Cruz, Santa Cruz, CA, USA, Sc-1836, 1/200), rabbit anti-VEGFR-2 (Santa-Cruz, sc$504,1 / 200$ ), mouse or rabbit anti-GFAP (Dako, Glostrup, Denmark, 6F2, 1/1000) and mouse antineuN (Chemicon, MAB377,1/500). After 3 washes in PBS 1X, slices were incubated for $2 \mathrm{~h}$ at room temperature with secondary fluorophore-coupled antibodies against goat, mouse or rabbit. After 3 washes of 10 min in PBS 1X slices were mounted with Mowiol. For vascular density, sections were observed with a Leitz DMRB microscope (Leica, Wetzlar, Germany) equipped for fluorescent microscopy. Images were digitized by a $1392 \times 1040$ resolution cooled CCD camera (Cool Snap, Princeton Instrument, Trenton, NJ, USA) on a computer with Cool Snap software and transferred to Adobe Photoshop Elements (version 4) for image processing. For ZO-1, VEGF and VEGFR-2 immunostaining staining sections were observed using a confocal microscope (Zeiss 510 Meta, Göttingen, Germany) equipped with an x63 objective (oil, numeric opening 1.4). We used an argon laser (excitation 488, emission 505-530 nm) for alexa 488, a helium laser (excitation 543, emission 585-615 nm) for Texas red and a krypton-argon laser (excitation 647 nm, emission 660-700 $\mathrm{nm}$ ) for alexa 647. Images were collected sequentially to avoid cross-contamination between fluorochromes. A series of 15 optical sections was projected onto a single image plane and scanned at $1024 \times 1024$ pixel resolution.

\section{Quantification of vascular density}

We used the point-counting method to quantify and compare the vascular density under different conditions [23]. This method has been already validated in human tissue, in vivo and in organotypic cultures [20-22]. It takes into account the number, size and tortuosity of vessels to characterize pathological angiogenesis. Briefly, a $5 \times 5$ grid was superposed onto the digitized image and the number of labeled vessels crossing the grid lines was counted. The score was expressed in arbitrary units of vascular density for a $1 \mathrm{~mm}^{2}$ area. Statistical analysis was performed: oneway analysis of variance (ANOVA) followed by Fisher test for $\mathrm{OHCs}(p<0.05$ is significant).

\section{Quantification of branching}

To evaluate the branching after each treatment, we selected magnifications of $0.5 \mathrm{~mm}^{2}$ areas in the two main hippocampal fields: CA1 and CA3. Vessel branch points resulting from microvascular sprouting [24], were counted manually and results were expressed as a percent of controls. Statistical analysis was performed by one-way analysis of variance (ANOVA) followed by Fisher test for $\mathrm{OHCs}(p<0.05$ is significant).

\section{Protein expression and activation}

Proteins were prepared according to our previous publication [21]. Protein samples $(40 \mu \mathrm{g})$ boiled in Laemmli buffer containing 2- $\beta$-mercaptoethanol were loaded onto a NupageNovex 4-12\% Bis-Tris Midi gel (Life Technologies), separated electrophoretically and transferred to polyvinyldifluoridine membranes (Hybond-C-extra, Amersham Biosciences, UK). Membranes were incubated overnight at $4^{\circ} \mathrm{C}$ with primary antibodies raised against VEGF (Santa Cruz, Sc-1836 1/200), VEGFR-2 (Abcam, Cambridge, MA, USA, Ab2349 1/1000), VEGFR-2P (Y1054 \& Y1059) (Abcam, Ab5473 1/1000), ZO-1 (Zymed, 61-7300 1/800), claudin-5 (Life Technologies, 34-1600, 1/400), occludin (Life Technologies, 71-1500, 1/500), or actin (LabVision, Fremont, CA, USA, ACTN05 1/1000), then with HRP secondary antibodies against rabbit, goat or mouse IgG for $1 \mathrm{~h}$ at RT. Bands were visualised by chemoluminescence detection (Western Lightening, Perkin Elmer, MA, USA). Western blots were analyzed by densitometry using Photoshop and Image and normalized with actin. Statistical analysis was performed by Kruskall-Wallis test, $\mathrm{p}<0.05$ is significant.

\section{Cytokine array}

The profile of cytokines released into the culture medium was analyzed by proteome profiler using rat cytokine array (R\&D Systems, Minneapolis, MN, USA, \# ARY008) according to the manufacturer's protocol. Results were analyzed by densitometry using ImageJ. For heatmap analysis, a difference between optical density of control slices and treatment conditions was calculated. Then the heatmap was generated using the MeV software (Boston, MA, USA).

\section{Results}

Organotypic cultures: a tool to decipher the mechanisms of BBB failure in epileptic disease

Since empty vessels respond to angiogenic factors, we studied the vascular remodelling after epileptic seizures using OHCs $[20,25,26]$. Previously, we observed the presence of angiogenesis and BBB permeability in pharmacoresistant temporal lobe epilepsies [22]. To determine if vascular remodeling was induced by seizures per se, or by cell damage or inflammation associated with severe seizures, we evaluated the vascular density in the 
following conditions: 1) seizures without damage induced by bicuculline; 2) inflammation induced by LPS; 3) seizures with neuronal death and inflammation induced by kainate.

To evaluate pathological angiogenesis, we measured vascular density and branching (Figure 1A). As a positive control, we also evaluated OHCs treated with VEGF. For the "point-counting" method, the number of "crossings" was counted and normalized to slice surface area in $\mathrm{mm}^{2}$. The "branch point" was evaluated after magnifying the slices (Figure 1B, C). We evaluated the branching and the vascular density $24 \mathrm{~h}$ post-treatment. A significant increase in vascular density was observed in all conditions indicating that seizures and also inflammation are sufficient to induce a vascular remodeling, $p<0.01$ compared to control (Figure 1D, E). However laminin staining showed an increased vascular network in CA1 and CA3 areas after kainate treatment compared to other conditions (Figure 1D). Neuronal death also occurred in these two areas after kainate treatment [27]. We quantified the branching in CA1

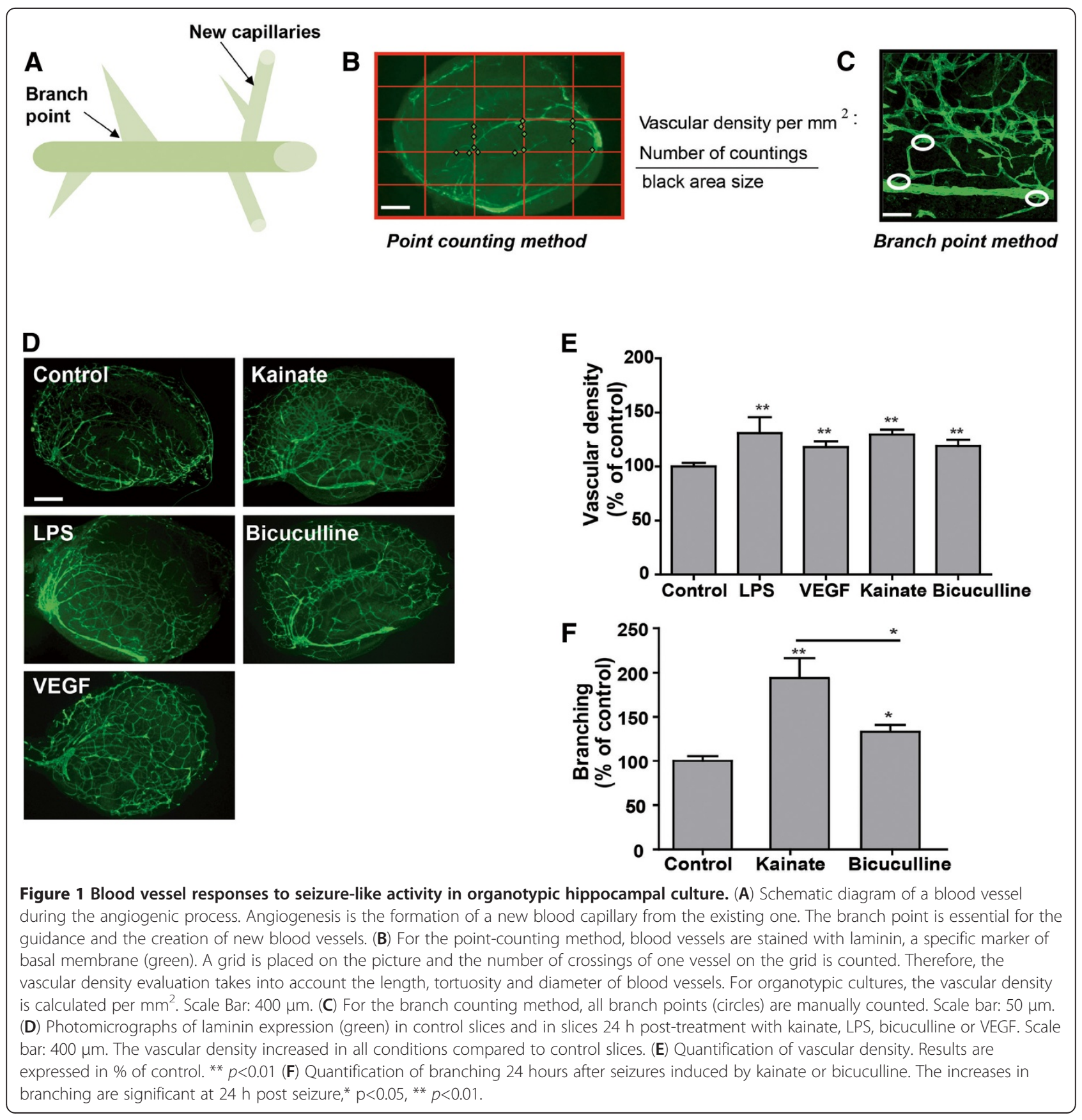


and CA3 areas $24 \mathrm{~h}$ after kainate and bicuculline treatments. An increase of branching was observed in all conditions. Interestingly the branching was significantly higher in the case of seizures associated with neuronal death (kainate) than seizures alone (bicuculline), $p<0.01$ and 0.05 , respectively, compared to control (Figure 1F).

We found an increase in vascular density and branching after in vitro seizures or inflammation, similar to previous results from rodent models or human tissue $[21,22]$. However, depending on the presence or absence of neuronal death, the vascular remodeling appeared to be different with an increase in branching in lesion areas. In the following study we compared changes in the NVU between the kainate and bicuculline models.

\section{Roles and modifications of NVU cells in pathological conditions}

The NVU is mainly composed of endothelial cells characterized by limited transport due to the presence of transporters and TJs. In the brain microvasculature, cells

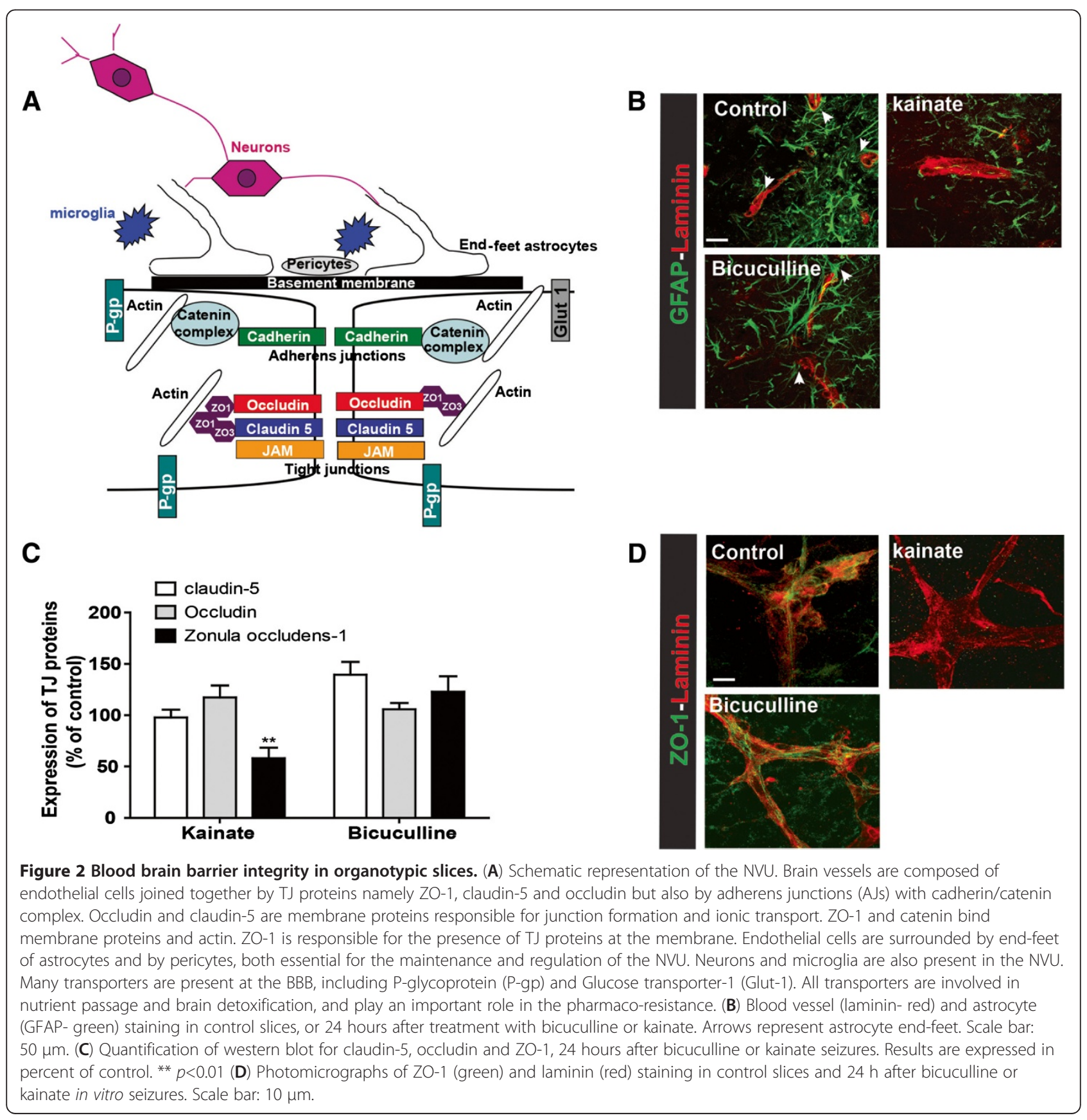



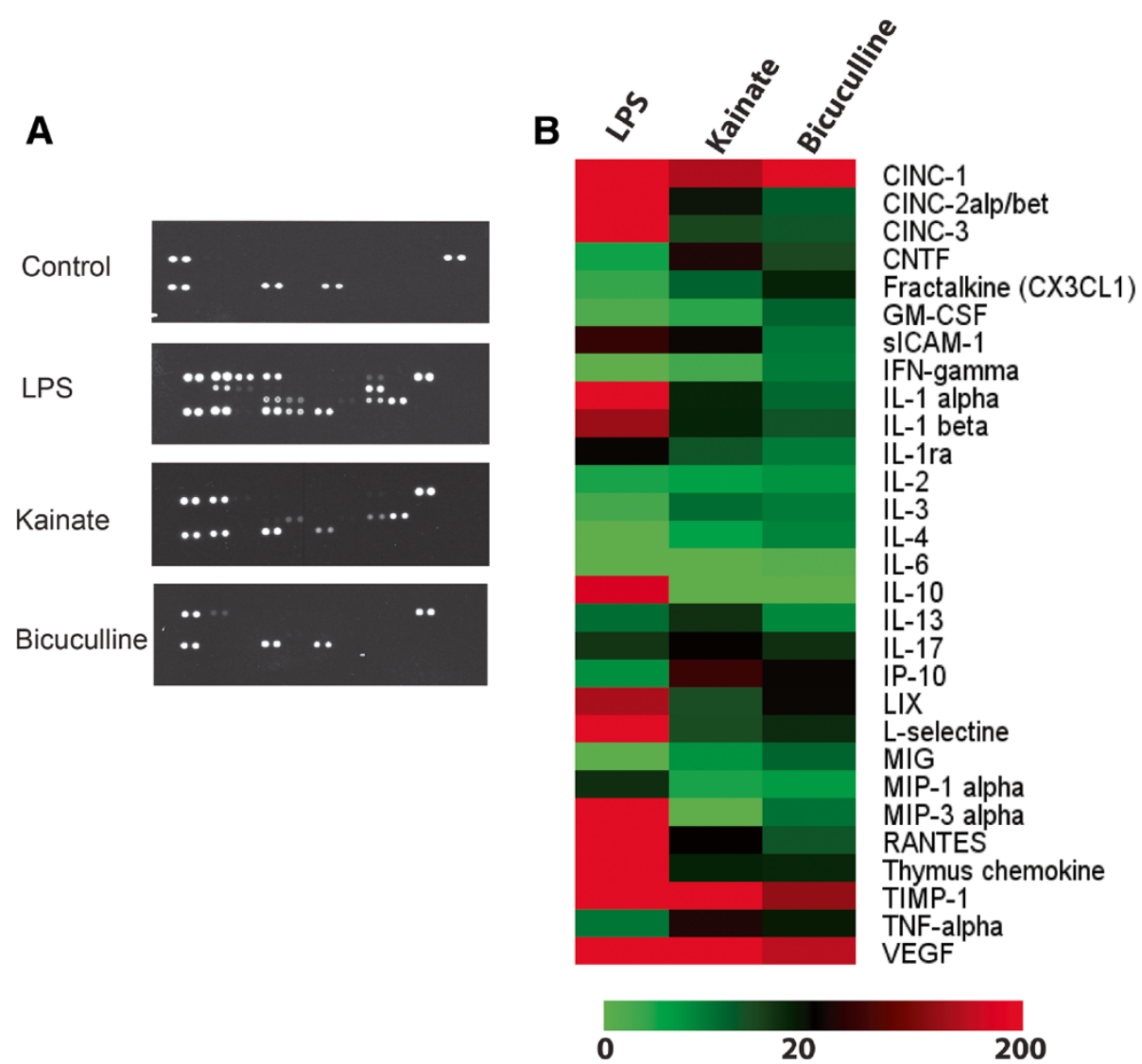

Figure 3 Organotypic slices are accessible for molecular screening in culture medium. (A) Membranes representing the cytokine microarray performed on culture medium $24 \mathrm{~h}$ after LPS, bicuculline or kainate treatment. Control corresponds to the culture medium of organotypic slices without treatment. (B) Heatmaps representing the optical density for each cytokine. Green represents low expression whereas red corresponds to high expression.

surrounding the capillaries include astrocytes and pericytes. These cells play strategic roles in both formation and maintenance of the NVU, and also in neurovascular coupling [1,9,28-30] (Figure 2A). The presence of astrocyte end-feet along and surrounding blood vessels was demonstrated by GFAP staining in control organotypic cultures (Figure 2B, arrows). The presence of these cells around blood vessels is modified in pathological conditions. Indeed, we observed a reduction of astrocyte end-feet $24 \mathrm{~h}$ after kainate treatment. However, after seizures without lesions, end-feet appeared intact (Figure 2B, arrows).

The main characteristic of the NVU is the presence of TJ proteins which scaffold the junctions between endothelial cells. These proteins are essential for a high transendothelial electrical resistance (TEER) (Figure 2A) [29,31]. The three important TJ proteins, ZO- 1 , claudin -5 and occludin, are preserved in culture for several weeks $[21,32]$.

We studied the regulation of ZO- 1 , claudin- 5 and occludin $24 \mathrm{~h}$ post-seizure induced by either kainate or bicuculline. In the kainate model, Western blot analysis revealed a significant down-regulation only for ZO-1, $p<0.01$. In the bicuculline model the expression of the three main TJ proteins was not affected (Figure 2C). The staining of ZO-1 and laminin revealed a regular staining of the TJ proteins along blood vessels in control slices. Similar staining was observed $24 \mathrm{~h}$ post-bicuculline seizures. However, at $24 \mathrm{~h}$ post-kainate seizures, ZO-1 staining was absent along blood vessels (Figure 2D). This experiment on OHCs showed that NVU remodeling is dependent on severity of neuronal damage induced by epileptiform stimuli.

OHCs are accessible for molecule screening in the culture medium

The tissue or culture medium from $\mathrm{OHCs}$ can be analysed by molecular screening techniques. Due to differences in branching and the regulation of NVU between two seizure models, we can expect differences in the secretion and release of angiogenic factors or cytokines. With a protein array, we evaluated the levels of secreted cytokines $24 \mathrm{~h}$ after seizures induced by kainate or bicuculline. As negative and positive controls, we used the medium of non-treated slices and the medium of $\mathrm{OHCs}$ treated with LPS. In the medium from non-treated slices, 
Table 1 Role of cytokines in inflammation, angiogenesis and BBB permeability

\begin{tabular}{|c|c|c|c|c|c|}
\hline Cytokines & Others name & Inflammation & Angiogenesis & BBB disruption & References \\
\hline \multirow[t]{2}{*}{ CINC-1 } & CXCL-1 or Gro-a & Pro & Pro & I & [34] \\
\hline & & & & & {$[35]$} \\
\hline \multirow[t]{2}{*}{ CINC-2 } & CXCL-3 or Gro-g & Pro & Pro & I & {$[35]$} \\
\hline & & & & & {$[36]$} \\
\hline \multirow[t]{2}{*}{ CINC-3 } & CXCL-2 or Gro-B & Pro & Pro & I & {$[35]$} \\
\hline & & & & & {$[37]$} \\
\hline CNTF & & Pro & I & I & {$[38]$} \\
\hline \multirow[t]{2}{*}{ Fractaline } & CX3CL-1 & Pro & Pro & I & [39] \\
\hline & & & & & [40] \\
\hline \multirow[t]{3}{*}{ GM-CSF } & & Pro & Pro & Pro & {$[40]$} \\
\hline & & & & & [41] \\
\hline & & & & & {$[42]$} \\
\hline S-ICAM-1 & CD54 & l & l & l & \\
\hline \multirow[t]{3}{*}{ IFN-g } & & Pro & Anti & Pro & [43] \\
\hline & & & & & [41] \\
\hline & & & & & [44] \\
\hline \multirow[t]{3}{*}{ IL1-a } & & Pro & Pro & Pro & [43] \\
\hline & & & & & [45] \\
\hline & & & & & {$[46]$} \\
\hline \multirow[t]{3}{*}{ IL1-b } & & Pro & Pro & Pro & {$[45]$} \\
\hline & & & & & [47] \\
\hline & & & & & {$[48]$} \\
\hline IL1-ra & & Anti & Anti & I & [49] \\
\hline \multirow[t]{2}{*}{ IL-2 } & & Pro & Pro & Pro & [50] \\
\hline & & & & & [51] \\
\hline IL3 & & & Pro & I & {$[52]$} \\
\hline \multirow[t]{3}{*}{ IL4 } & & Pro & Anti & Pro & [50] \\
\hline & & & & & [51] \\
\hline & & & & & [35] \\
\hline \multirow[t]{3}{*}{ IL6 } & & Pro and anti & Pro & Pro & {$[35]$} \\
\hline & & & & & {$[48]$} \\
\hline & & & & & [53] \\
\hline IL10 & & Anti & Anti & I & [54] \\
\hline IP-10 & CXCL10 & Pro & Anti & I & [35] \\
\hline LIX & CXCL5 & Pro & Pro & I & [35] \\
\hline L-selectine & CD62L & l & I & I & \\
\hline MIG & CXCL9 & Pro & Anti & I & [35] \\
\hline MIP-1a & $\mathrm{CCL} 3$ & Pro & I & I & [35] \\
\hline MIP-3a & CCL20 & Pro & I & I & [55] \\
\hline RANTES & CCL5 & Pro & Anti & Pro & {$[56]$} \\
\hline TIMP-1 & & I & Anti & I & [57] \\
\hline \multirow[t]{2}{*}{ TNF-a } & & Pro & Pro & Pro & {$[48]$} \\
\hline & & & & & [35] \\
\hline VEGF & & l & Pro & Pro & [58] \\
\hline
\end{tabular}



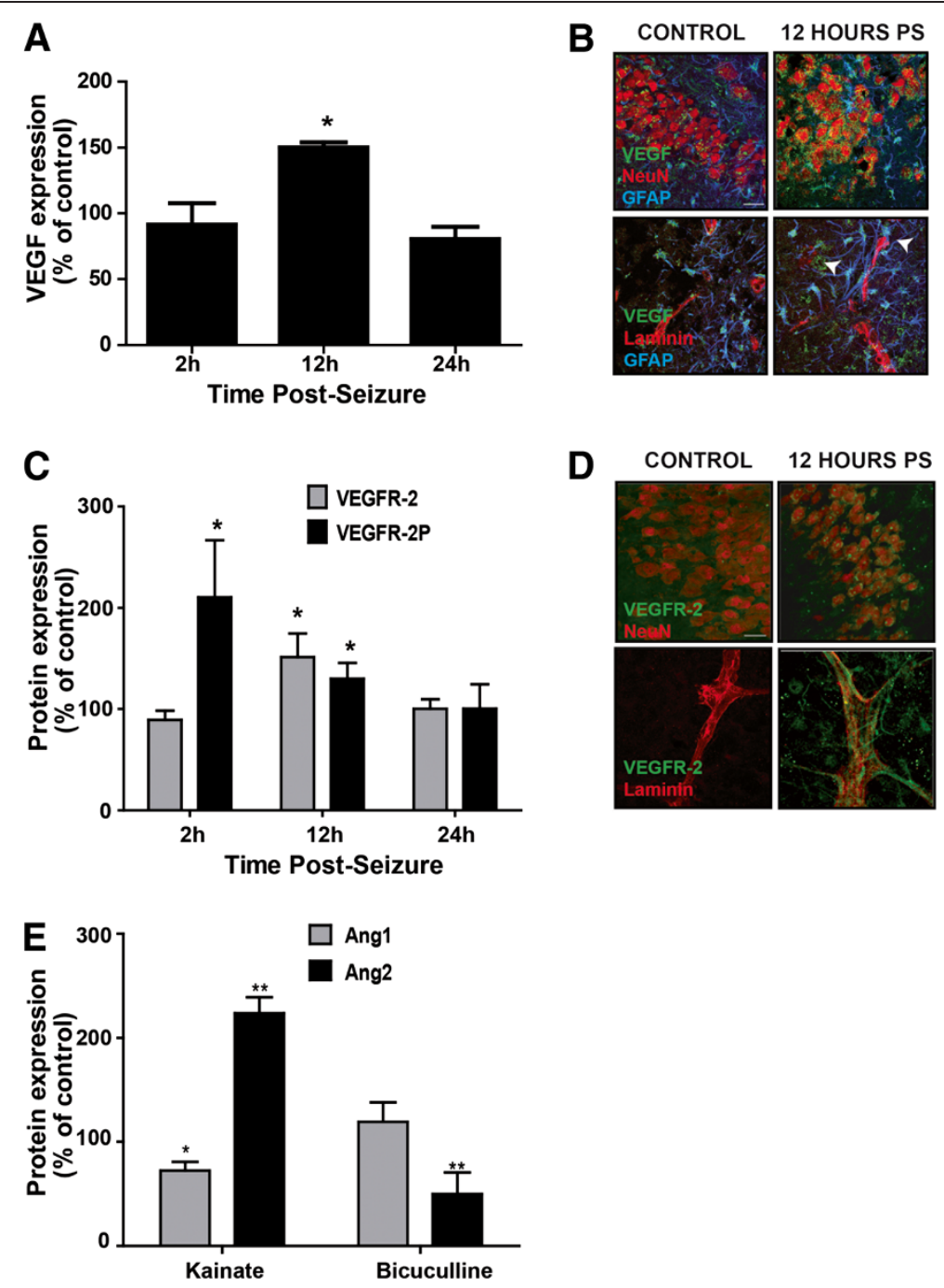

Figure 4 Organotypic slices are accessible for analysis of proteins in tissue. (A) Analysis of VEGF expression by Western blot 2, 12 and $24 \mathrm{~h}$ post-bicuculline treatment. Results are expressed in percent of control, * $p<0.05$. (B) Immunostaining of VEGF (green), NeuN or laminin (red) and GFAP (blue) in control slices or $12 \mathrm{~h}$ post-bicuculline seizures (PS). VEGF is expressed in neurons and in astrocytes around blood vessels (arrows), Scale bar $10 \mu \mathrm{m}$. (C) Analysis of VEGFR-2 expression and activation by Western blot 2, 12 and $24 \mathrm{~h}$ post-bicuculline treatment. Results are expressed in percent of control, ${ }^{*} p<0.05$. (D) Immunostaining of VEGFR-2 (green), NeuN or laminin (red) in control slices or $12 \mathrm{~h}$ post-bicuculline seizures. VEGFR-2 is expressed in neurons and along blood vessels $12 \mathrm{~h}$ after bicuculline treatment. Scale bar $10 \mu \mathrm{m}$. (E) Analysis of Ang1 and Ang2 expression by Western blot $24 \mathrm{~h}$ post-bicuculline or kainate treatment. Results are expressed in percent of control, * $p<0.05,{ }^{* *} p<0.01$.

we found only 2 cytokines: VEGF and metallopeptidase inhibitor-1 (TIMP)-1 while $24 \mathrm{~h}$ after LPS treatment, many additional cytokines were secreted into the culture medium. After seizure induction, we observed different patterns between kainate and bicuculline models. In kainate model, cytokines present in the culture medium indicated an inflammatory process, confirming previous results $[27,33]$. In contrast, $24 \mathrm{~h}$ after bicuculline seizures, cytokine patterns were identical to those in control slices (Figure 3A). These differences in cytokine profiles were confirmed by heatmap analysis. We focused in more detail on cytokines known to be involved in vascular remodeling by inducing angiogenesis or increased BBB permeability.
VEGF, IL-1 $\beta$, IL-1 $\alpha$, IL-6, IL-13 and also TNF- $\alpha$ are proangiogenic, while IFN- $\gamma$, IL-1 $\beta$ and VEGF can also participate in BBB permeability (Table 1). Most of these cytokines, including IL-1 $\alpha$, IL1- $\beta$ but also VEGF, are present at higher levels in the kainate compared to bicuculline model (Figure 3B).

\section{Protein analysis in tissue}

Angiogenesis and $\mathrm{BBB}$ permeability are hallmarks of VEGF/VEGFR-2 activation [59]. Their overexpression in the epileptic focus after experimental seizures suggests that the VEGF/VEGFR-2 system is a logical new target for refractory epilepsies $[21,22,60,61]$. In the kainate model, we 
A
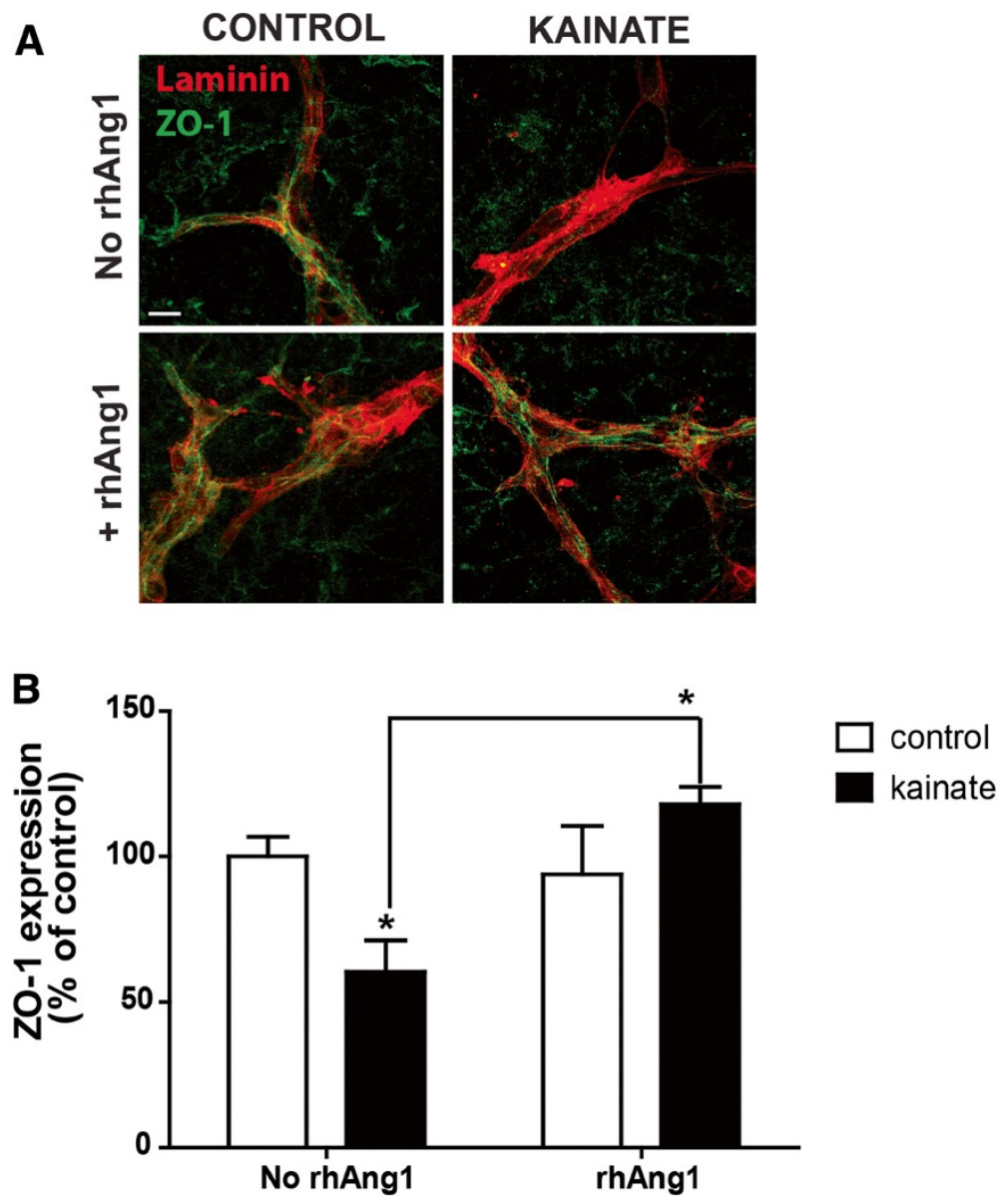

Figure 5 Organotypic slices are accessible for drugs testing. (A) Immunostaining of ZO-1 (green) and laminin (red) in control slices and $24 \mathrm{~h}$ post-kainate seizures with or without rh-Ang1 application $4 \mathrm{~h}$ post-seizures. Rh-Ang-1 repaired the loss of ZO-1 (green) in blood vessels (laminin, red). Scale bar: $50 \mu \mathrm{m}$ (B) Analysis of Zonula occludens-1 expression by Western blot $24 \mathrm{~h}$ post-kainate treatment with or without rhAng1 application. Results are expressed in percent of control, ${ }^{*} p<0.05$.

demonstrated an upregulation and activation of VEGF/ VEGFR-2 signaling, leading to the downregulation of ZO-1 [21]. The lower level of VEGF in the culture medium of $\mathrm{OHCs}$ after bicuculline treatment suggested a different regulation of angiogenic factors that does not trigger a loss of $\mathrm{TJ}$ proteins.

Western blotting and immunostaining demonstrated an overexpression of VEGF only $12 \mathrm{~h}$ after bicuculline application, $p<0.05$ (Figure 4A). Increased VEGF was observed in astrocytic end-feet and surrounding blood vessels (arrows, Figure 4B). We next studied the expression and activation of VEGFR-2. An increase in VEGFR-2 expression was also detected $12 \mathrm{~h}$ post-seizure, $p<0.05$. However, the activation of VEGFR-2, measured by the phosphorylation of the receptor, was visible at 2 and $12 \mathrm{~h}$ post-seizure, $p<0.05$ for both (Figure $4 \mathrm{C}$ ). The immunostaining revealed an overexpression of the receptor in neurons but more particularly in blood vessels (Figure 4D).
After bicuculline-induced seizures, we observed a similar upregulation of the VEGF/VEGFR-2 system.

To understand differences in the regulation of tight junctions between the two seizure models, we focused on the angiopoietin system, composed of angiopoietin-1 and 2 (Ang1 and Ang2). These two proteins have opposite effects on the BBB integrity; Ang1 is involved in the maturation of blood vessels and participates in the $\mathrm{BBB}$ integrity, whereas Ang2 appears in early stages of the angiogenesis and disrupts the BBB [62-65]. We decided to study the regulation of these two proteins $24 \mathrm{~h}$ after either kainate or bicuculline seizures, a time-point where ZO-1 is downregulated in the kainate model only. By Western blotting, we observed the same level of Ang1 as in control slices but a significant downregulation of Ang2 following bicuculline seizures, $p<0.01$. However, after kainate seizures, Ang1 expression was significantly lower than in control slices, $p<0.05$, whereas Ang2 was 
significantly upregulated, $p<0.01$ (Figure 4E). These results suggest that angiopoietin system could play an important role in the regulation of $\mathrm{TJ}$ proteins after epileptic seizures.

\section{Drug testing and molecular screening}

Due to their easy accessibility, OHCs are excellent tools for pharmacological and biochemical assays, including drug screening for $\mathrm{BBB}$ protective compounds that could improve the treatments for ischemic or traumatic injuries $[66,67]$. We have already demonstrated the use of neutralizing antibodies in OHCs. We neutralized VEGF with an anti-VEGF antibody (bevacizumab) that prevents VEGF binding to its receptor. Despite the thickness of the culture plus insert at around $150 \mu \mathrm{m}$, the addition of this neutralizing antibody abolished both the down-regulation of ZO-1 protein and increased vascularisation induced by in vitro seizures [21]. Since in this study a deregulation of the Ang proteins was observed only in the model where ZO-1 was also downregulated, we tested the effect of recombinant Angiopoietin-1 (rhAng-1) applied to the culture medium. To determine if we can restore $\mathrm{ZO}-1$ expression after kainate seizures, rhAng1 was added to the culture medium $4 \mathrm{~h}$ post-seizures. In control slices treated with rhAng1, the staining pattern of ZO-1 along blood vessels did not change compared to control slices. Treatment with rhAng1 after kainate seizures restored the presence of ZO-1 protein (Figure 5A). Western blot analysis confirmed the above results, showing a significant increase of ZO-1 expression after rhAng1 addition, $p<0.05$, and confirming that Ang1 plays an important role in the restoration of BBB integrity (Figure 5B).

\section{Discussion}

Due to the presence of all cell types and their interactions, preservation of TJs between endothelial cells, as well as $\mathrm{BBB}$ carriers and transporters, brain slices provide a complete ex vivo model of the NVU, although in the absence of blood flow. For example, we have observed that NVU alterations in OHSc, including increased vascularisation and TJ disassembly, were similar to those reported in human in vivo focal epilepsy. Other cell types present at the BBB can be studied in OHCs. Indeed, a recent paper focused on the intact functions of NVU in OHCs, showing that calcium signaling can be investigated in astrocyte end-feet and that the contractile properties of pericytes, necessary for vasomodulation, are conserved for weeks in culture $[11,68]$. Moreover, microglia and neurons are also present in the NVU, but the role of microglia in NVU regulation is still not clear and poorly studied in organotypic cultures. The only link between microglia and blood vessels was the presence of active microglia surrounding blood vessels in retinal organotypic cultures [69]. Finally, the presence of transporters such as glucose transporter-1 and P-glycoprotein on brain endothelial cells in organotypic slices has been previously documented. Moreover, it has been shown that the transport function of P-gp, involved in the pharmacoresistance of several neurological diseases, is still preserved in organotypic slices [30].

Organotypic slices are also useful for testing the ability of different drugs to affect/protect the NVU, such as inhibitors of signaling pathways and neutralizing antibodies [21]. In this study we showed that NVU integrity was restored by the application of recombinant Ang1. BBB dysfunction in diverse CNS disorders, including epilepsy, Alzheimer's disease and ischemia, is in part due to the loss of AJ or TJ proteins along microvessels [21,22,70,71].

However, a limitation of organotypic cultures is the lack of tools to estimate BBB permeability, which is altered in several brain pathologies [22,72-74]. Staining for serum protein leakage or the measurement of TEER in vitro [75] is not possible in this model. To counteract this issue, a co-culture model of endothelial cells and brain slices has been developed in which Duport and colleagues showed that the BBB permeability can be evaluated by microdialysis [76].

In the past decade, alterations in the neuro-vasculature have been shown to be important in many CNS diseases, including glioma, stroke, Alzheimer's disease and epilepsy [2-5,77]. However the mechanisms of NVU dysregulation are still unknown in several pathologies. Using organotypic slice cultures to study NVU embedded in the microenvironment of anatomically organized parenchymal cells and maintaining many important physiological functions, will undoubtedly facilitate future studies on mechanisms and impact of pathological conditions on NVU remodeling as well as its role in disease processes.

\section{Abbreviations}

AJs: Adherent junctions; Ang1: Angiopoeitin-1; Ang2: Angiopoeitin-2; BBB: Blood-brain Barrier; NVU: Neuro-vascular Unit; OHCs: Organotypic hippocampal cultures; rhAng1: Recombinant humain Angiopoeitin-1; rrVEGF: Recombinant rat VEGF; TEER: Transendothelial electric resistance; TJs: Tight junction proteins; TLE: Temporal lobe epilepsy; VEGF: Vascular endothelial growth factor; VEGFR-2: Vascular endothelial growth factor receptor-2; ZO-1: Zonula occludens-1.

\section{Competing interests}

The authors declare that they have no competing interests.

\section{Authors' contributions}

$M M B, F d B, M L N$ designed research. FdB performed organotypic hippocampal slices preparation. MMB performed immunostaining and quantified vascularization. MMB and MLN wrote the paper. All authors read and approved the final manuscript.

\section{Acknowledgments}

The authors are indebted to the Agence Nationale de la Recherche ANR MNP 2009, for supporting this research project.

\section{Author details}

${ }^{1}$ Institut de Génomique Fonctionnelle, CNRS UMR5203, INSERM U661, Université Montpellier 1, 2, Montpellier, France. ${ }^{2}$ Department of Neurosurgery, Thomas Jefferson University, 1020 Locust Street, JAH 454 Philadelphia, PA 19107, USA. 
Received: 19 September 2012 Accepted: 28 January 2013

Published: 7 February 2013

\section{References}

1. Abbott NJ, Ronnback L, Hansson E: Astrocyte-endothelial interactions at the blood-brain barrier. Nat Rev Neurosci 2006, 7:41-53.

2. Bennett J, Basivireddy J, Kollar A, Biron KE, Reickmann P, Jefferies WA, McQuaid S: Blood-brain barrier disruption and enhanced vascular permeability in the multiple sclerosis model EAE. J Neuroimmunol 2010, 229:180-191.

3. Friedman A: Blood-brain barrier dysfunction, status epilepticus, seizures, and epilepsy: a puzzle of a chicken and egg? Epilepsia 2011, 52(Suppl 8):19-20.

4. Tomkins O, Feintuch A, Benifla M, Cohen A, Friedman A, Shelef I: Bloodbrain barrier breakdown following traumatic brain injury: a possible role in posttraumatic epilepsy. Cardiovasc Psychatry Neurol 2011, 2011:765923.

5. Ujiie M, Dickstein DL, Carlow DA, Jefferies WA: Blood-brain barrier permeability precedes senile plaque formation in an Alzheimer disease model. Microcirculation 2003, 10:463-470.

6. Naik P, Cucullo L: In vitro blood-brain barrier models: current and perspective technologies. J Pharm Sci 2012, 101:1337-1354.

7. Wilhelm I, Fazakas C, Krizbai IA: In vitro models of the blood-brain barrier. Acta neurobiolExp (Wars) 2011, 71:113-128.

8. Silbergeld DL, Ali-Osman F: Isolation and characterization of microvessels from normal brain and brain tumors. J Neurooncol 1991, 11:49-55.

9. Hamm S, Dehouck B, Kraus J, Wolburg-Buchholz K, Wolburg H, Risau W, Cecchelli R, Engelhardt B, Dehouck MP: Astrocyte mediated modulation of blood-brain barrier permeability does not correlate with a loss of tight junction proteins from the cellular contacts. Cell Tissue Res 2004, 315:157-166.

10. Hori S, Ohtsuki S, Tachikawa M, Kimura N, Kondo T, Watanabe M, Nakashima $E$, Terasaki T: Functional expression of rat $A B C G 2$ on the luminal side of brain capillaries and its enhancement by astrocyte-derived soluble factor (s). J Neurochem 2004, 90:526-536.

11. Dore-Duffy P, Cleary K: Morphology and properties of pericytes. Methods Mol Biol 2011, 686:49-68.

12. Al Ahmad A, Taboada CB, Gassmann M, Ogunshola OO: Astrocytes and pericytes differentially modulate blood-brain barrier characteristics during development and hypoxic insult. J Cereb Blood Flow 2011, 31:693-705

13. Cucullo L, Marchi N, Hossain M, Janigro D: A dynamic in vitro BBB model for the study of immune cell trafficking into the central nervous system. J Cereb Blood Flow 2011, 31:767-777.

14. Hallier-Vanuxeem D, Prieto P, Culot M, Diallo H, Landry C, Tahti H, Cecchell R: New strategy for alerting central nervous system toxicity: Integration of blood-brain barrier toxicity and permeability in neurotoxicity assessment. Toxicol in vitro 2009, 23:447-453.

15. Hatherell K, Couraud PO, Romero IA, Weksler B, Pilkington GJ: Development of a three-dimensional, all-human in vitro model of the blood-brain barrier using mono-, co-, and tri-cultivation Transwell models. J Neurosci Methods 2011, 199:223-229.

16. Gahwiler BH: Organotypic monolayer cultures of nervous tissue. J Neurosci Methods 1981, 4:329-342.

17. Stoppini L, Buchs PA, Muller D: A simple method for organotypic cultures of nervous tissue. J Neurosci Methods 1991, 37:173-182.

18. Del Turco D, Deller T: Organotypic entorhino-hippocampal slice culturesa tool to study the molecular and cellular regulation of axonal regeneration and collateral sprouting in vitro. Methods Mol Biol 2007, 399:55-66.

19. Gahwiler BH, Capogna M, Debanne D, McKinney RA, Thompson SM: Organotypic slice cultures: a technique has come of age. Trends Neurosci 1997, 20:471-477.

20. Moser KV, Humpel C: Vascular endothelial growth factor counteracts NMDA-induced cell death of adult cholinergic neurons in rat basal nucleus of Meynert. Brain Res Bull 2005, 65:125-131.

21. Morin-Brureau M, Lebrun A, Rousset MC, Fagni L, Bockaert J, de Bock F, Lerner-Natoli M: Epileptiform activity induces vascular remodeling and ZO-1 downregulation in organotypic hippocampal cultures: role of VEGF signaling pathways. J Neurosci 2011, 31:10677-10688.

22. Rigau V, Morin M, Rousset MC, de Bock F, Lebrun A, Coubes P, Picot MC, Baldy-Moulinier M, Bockaert J, Crespel A, Lerner-Natoli M: Angiogenesis is associated with blood-brain barrier permeability in temporal lobe epilepsy. Brain 2007, 130:1942-1956.

23. de Paz P, Barrio JP: Stereological parameters from the analysis of the cell micrographs either by manual point-counting methods or by using a semi-automatic system: a BASIC program for ZX-Spectrum personal computer. Comput Biol Med 1985, 15:153-158.

24. Horowitz A, Simons M: Branching morphogenesis. Cir Res 2008, 103:784-795.

25. Poulsen FR, Jahnsen $H$, Blaabjerg M, Zimmer J: Pilocarpine-induced seizure-like activity with increased BNDF and neuropeptide $Y$ expression in organotypic hippocampal slice cultures. Brain Res 2002, 950:103-118.

26. Ziobro JM, Deshpande LS, Delorenzo RJ: An organotypic hippocampal slice culture model of excitotoxic injury induced spontaneous recurrent epileptiform discharges. Brain Res 2011, 1371:110-120.

27. de Bock F, Derijard B, Dornand J, Bockaert J, Rondouin G: The neuronal death induced by endotoxic shock but not that induced by excitatory amino acids requires TNF-alpha. Eur J Neurosci 1998, 10:3107-3114.

28. Abbott NJ: Astrocyte-endothelial interactions and blood-brain barrier permeability. J Anat 2002, 200:629-638

29. Abbott NJ, Patabendige AA, Dolman DE, Yusof SR, Begley DJ: Structure and function of the blood-brain barrier. Neurobiol Dis 2010, 37:13-25.

30. Wolburg H, Noell S, Mack A, Wolburg-Buchholz K, Fallier-Becker P: Brain endothelial cells and the glio-vascular complex. Cell Tissue Res 2009, 335:75-96.

31. Correale J, Villa A: Cellular elements of the blood-brain barrier. Neurochem Res 2009, 34:2067-2077.

32. Bendfeldt K, Radojevic V, Kapfhammer J, Nitsch C: Basic fibroblast growth factor modulates density of blood vessels and preserves tight junctions in organotypic cortical cultures of mice: a new in vitro model of the blood-brain barrier. J Neurosci 2007, 27:3260-3267.

33. Jarvela JT, Ruohonen S, Kukko-Lukjanov TK, Plysjuk A, Lopez-Picon FR, Holopainen IE: Kainic acid-induced neurodegeneration and activation of inflammatory processes in organotypic hippocampal slice cultures: treatment with cyclooxygenase- 2 inhibitor does not prevent neuronal death. Neuropharmacology 2011, 60:1116-1125.

34. Barichello T, Generoso JS, Silvestre C, Costa CS, Carrodore MM, Cipriano AL, Michelon CM, Petronilho F, Dal-Pizzol F, Vilela MC, Teixeira AL: Circulating concentrations, cerebral output of the CINC-1 and blood-brain barrier disruption in Wistar rats after pneumococcal meningitis induction. Eur J Clin Microbiol Infect Dis 2012, 31:2005-2009.

35. Keeley EC, Mehrad B, Strieter RM: Chemokines as mediators of tumor angiogenesis and neovascularization. Exp Cell Res 2011, 317:685-690

36. Ahuja SK, Murphy PM: The CXC chemokines growth-regulated oncogene (GRO) alpha, GRObeta, GROgamma, neutrophil-activating peptide-2, and epithelial cell-derived neutrophil-activating peptide-78 are potent agonists for the type $B$, but not the type $A$, human interleukin- 8 receptor. J Biol Chem 1996, 271:20545-20550.

37. Wolpe SD, Sherry B, Juers D, Davatelis G, Yurt RW, Cerami A: Identification and characterization of macrophage inflammatory protein 2. Proc Natl Acad Sci U S A 1989, 86:612-616.

38. Linker R, Gold R, Luhder F: Function of neurotrophic factors beyond the nervous system: inflammation and autoimmune demyelination. Crit Rev Immunol 2009, 29:43-68.

39. Bernardini G, Ribatti D, Spinetti G, Morbidelli L, Ziche M, Santoni A, Capogrossi MC, Napolitano M: Analysis of the role of chemokines in angiogenesis. J Immunol Methods 2003, 273:83-101.

40. Pan Y, Lloyd C, Zhou H, Dolich S, Deeds J, Gonzalo JA, Vath J, Gosselin M, Ma J, Dussault B, et al: Neurotactin, a membrane-anchored chemokine upregulated in brain inflammation. Nature 1997, 387:611-617.

41. Niu X, Wang H, Fu ZF: Role of chemokines in rabies pathogenesis and protection. Adv Virus Res 2011, 79:73-89.

42. Iwami K, Natsume A, Wakabayashi T: Cytokine networks in glioma. Neurosurg Rev 2011, 34:253-263. discussion 263-254.

43. Naldini A, Carraro F: Role of inflammatory mediators in angiogenesis. Curr Drug Targets Inflamm Allergy 2005, 4:3-8.

44. Schoenborn JR, Wilson CB: Regulation of interferon-gamma during innate and adaptive immune responses. Adv Immunol 2007, 96:41-101.

45. Benveniste EN: Inflammatory cytokines within the central nervous system: sources, function, and mechanism of action. Am J Physiol 1992, 263:C1-16.

46. Gloor SM, Weber A, Adachi N, Frei K: Interleukin-1 modulates protein tyrosine phosphatase activity and permeability of brain endothelial cells. Biochem Biophys Res Commun 1997, 239:804-809. 
47. Voronov E, Shouval DS, Krelin Y, Cagnano E, Benharroch D, Iwakura Y, Dinarello CA, Apte RN: IL-1 is required for tumor invasiveness and angiogenesis. Proc Natl Acad Sci U S A 2003, 100:2645-2650.

48. de Vries HE, Blom-Roosemalen MC, van Oosten M, de Boer AG, van Berkel TJ, Breimer DD, Kuiper J: The influence of cytokines on the integrity of the blood-brain barrier in vitro. J Neuroimmunol 1996, 64:37-43.

49. Bar D, Apte RN, Voronov E, Dinarello CA, Cohen S: A continuous delivery system of IL-1 receptor antagonist reduces angiogenesis and inhibits tumor development. FASEB J 2004, 18:161-163.

50. Banks WA, Erickson MA: The blood-brain barrier and immune function and dysfunction. Neurobiol Dis 2010, 37:26-32.

51. Bae J, Park D, Lee YS, Jeoung D: Interleukin-2 promotes angiogenesis by activation of Akt and increase of ROS. J Microbiol Biotechnol 2008, 18:377-382.

52. Dentelli P, Del Sorbo L, Rosso A, Molinar A, Garbarino G, Camussi G, Pegoraro L, Brizzi MF: Human IL-3 stimulates endothelial cell motility and promotes in vivo new vessel formation. J Immunol 1999, 163:2151-2159.

53. Scheller J, Chalaris A, Schmidt-Arras D, Rose-John S: The pro- and antiinflammatory properties of the cytokine interleukin-6. Biochim Biophys Acta 2011, 1813:878-888

54. Pestka S, Krause CD, Sarkar D, Walter MR, Shi Y, Fisher PB: Interleukin-10 and related cytokines and receptors. Annu Rev Immunol 2004, 22:929-979.

55. Rossi DL, Vicari AP, Franz-Bacon K, McClanahan TK, Zlotnik A: Identification through bioinformatics of two new macrophage proinflammatory human chemokines: MIP-3alpha and MIP-3beta. J Immunol 1997, 158:1033-1036.

56. Terao S, Yilmaz G, Stokes KY, Russell J, Ishikawa M, Kawase T, Granger DN: Blood cell-derived RANTES mediates cerebral microvascular dysfunction, inflammation, and tissue injury after focal ischemia-reperfusion. Stroke 2008, 39:2560-2570.

57. Ikenaka Y, Yoshiji H, Kuriyama S, Yoshii J, Noguchi R, Tsujinoue H, Yanase K, Namisaki T, Imazu H, Masaki T, Fukui H: Tissue inhibitor of metalloproteinases-1 (TIMP-1) inhibits tumor growth and angiogenesis in the TIMP-1 transgenic mouse model. Int J Cancer 2003, 105:340-346.

58. Carmeliet $P$, Jain RK: Molecular mechanisms and clinical applications of angiogenesis. Nature 2011, 473:298-307.

59. Argaw AT, Asp L, Zhang J, Navrazhina K, Pham T, Mariani JN, Mahase S, Dutta DJ, Seto J, Kramer EG, et al: Astrocyte-derived VEGF-A drives blood-brain barrier disruption in CNS inflammatory disease. J Clin Invest 2012, 122:2454-2468.

60. Marcon J, Gagliardi B, Balosso S, Maroso M, Noe F, Morin M, Lerner-Natoli M, Vezzani A, Ravizza T: Age-dependent vascular changes induced by status epilepticus in rat forebrain: implications for epileptogenesis. Neurobiol Dis 2009, 34:121-132.

61. Nikitidou L, Kanter-Schlifke I, Dhondt J, Carmeliet P, Lambrechts D, Kokaia M: VEGF receptor-2 (flk-1) overexpression in mice counteracts focal epileptic seizures. PLoS One 2012, 7:e40535.

62. Nag S, Papneja T, Venugopalan R, Stewart DJ: Increased angiopoietin2 expression is associated with endothelial apoptosis and blood-brain barrier breakdown. Lab Invest 2005, 85:1189-1198.

63. Shen F, Walker EJ, Jiang L, Degos V, Li J, Sun B, Heriyanto F, Young WL, Su $\mathrm{H}$ : Coexpression of angiopoietin-1 with VEGF increases the structural integrity of the blood-brain barrier and reduces atrophy volume. J Cereb Blood Flow 2011, 31:2343-2351.

64. Yu H, Wang $P, A n P, X u e ~ Y$ : Recombinant human angiopoietin-1 ameliorates the expressions of ZO-1, occludin, VE-cadherin, and PKCalpha signaling after focal cerebral ischemia/reperfusion in rats. J Mol Neurosci 2012, 46:236-247.

65. Zhu Y, Lee C, Shen F, Du R, Young WL, Yang GY: Angiopoietin-2 facilitates vascular endothelial growth factor-induced angiogenesis in the mature mouse brain. Stroke 2005, 36:1533-1537.

66. Pena F: Organotypic cultures as tool to test long-term effects of chemicals on the nervous system. Curr Med Chem 2010, 17:987-1001.

67. Sundstrom L, Morrison B 3rd, Bradley M, Pringle A: Organotypic cultures as tools for functional screening in the CNS. Drug Discov Today 2005, 10:993-1000.

68. Kovacs R, Papageorgiou I, Heinemann U: Slice cultures as a model to study neurovascular coupling and blood brain barrier in vitro. Cardiovasc Psychiatry Neurol 2011, 2011:646958.

69. Mertsch K, Hanisch UK, Kettenmann H, Schnitzer J: Characterization of microglial cells and their response to stimulation in an organotypic retinal culture system. J Comp Neurol 2001, 431:217-227.
70. Biron KE, Dickstein DL, Gopaul R, Jefferies WA: Amyloid triggers extensive cerebral angiogenesis causing blood brain barrier permeability and hypervascularity in Alzheimer's disease. PLoS One 2011, 6:e23789.

71. Jiao $H$, Wang $Z$, Liu $Y$, Wang $P$, Xue $Y$ : Specific role of tight junction proteins claudin-5, occludin, and ZO-1 of the blood-brain barrier in a focal cerebral ischemic insult. J Mol Neurosci: MN 2011, 44:130-139.

72. Marchi N, Guiso G, Caccia S, Rizzi M, Gagliardi B, Noe F, Ravizza T, Bassanini S, Chimenti S, Battaglia G, Vezzani A: Determinants of drug brain uptake in a rat model of seizure-associated malformations of cortical development. Neurobiol Dis 2006, 24:429-442.

73. van Vliet EA, da Costa AS, Redeker S, van Schaik R, Aronica E, Gorter JA: Blood-brain barrier leakage may lead to progression of temporal lobe epilepsy. Brain 2007, 130:521-534.

74. Zlokovic BV: Neurodegeneration and the neurovascular unit. Nat Med 2010, 16:1370-1371.

75. Boveri M, Berezowski V, Price A, Slupek S, Lenfant AM, Benaud C, Hartung T, Cecchelli R, Prieto P, Dehouck MP: Induction of blood-brain barrier properties in cultured brain capillary endothelial cells: comparison between primary glial cells and C6 cell line. Glia 2005, 51:187-198.

76. Duport S, Stoppini L, Correges P: Electrophysiological approach of the antiepileptic effect of dexamethasone on hippocampal slice culture using a multirecording system: the Physiocard. Life Sci 1997, 60:PL 251-256.

77. Zlokovic BV: Neurovascular pathways to neurodegeneration in Alzheimer's disease and other disorders. Nat Rev Neurosci 2011, 12:723-738.

doi:10.1186/2045-8118-10-11

Cite this article as: Morin-Brureau et al:: Organotypic brain slices: a model to study the neurovascular unit micro-environment in epilepsies. Fluids and Barriers of the CNS 2013 10:11.

\section{Submit your next manuscript to BioMed Central and take full advantage of:}

- Convenient online submission

- Thorough peer review

- No space constraints or color figure charges

- Immediate publication on acceptance

- Inclusion in PubMed, CAS, Scopus and Google Scholar

- Research which is freely available for redistribution
C) Biomed Central 